Draft Version November 9, 2018

Preprint typeset using LATEX style emulateapj v. 5/25/10

\title{
THE OPTICALLY UNBIASED GRB HOST (TOUGH) SURVEY. III. REDSHIFT DISTRIBUTION
}

\author{
P. Jakobsson ${ }^{1}$, J. Hjorth ${ }^{2}$, D. Malesani ${ }^{2}$, R. Chapman ${ }^{1,3}$, J. P. U. Fynbo ${ }^{2}$, N. R. TanviR ${ }^{4}$, B. Milvang-Jensen $^{2}$, \\ P. M. Vreeswijk ${ }^{1}$, G. Letawe ${ }^{5}$, and R. L. C. Starling ${ }^{4}$ \\ Draft version November 9, 2018
}

\begin{abstract}
We present 10 new gamma-ray burst (GRB) redshifts and another five redshift limits based on host galaxy spectroscopy obtained as part of a large program conducted at the Very Large Telescope (VLT). The redshifts span the range $0.345 \leq z \lesssim 2.54$. Three of our measurements revise incorrect values from the literature. The homogeneous host sample researched here consists of 69 hosts that originally had a redshift completeness of 55\% (with 38 out of 69 hosts having redshifts considered secure). Our project, including VLT/X-shooter observations reported elsewhere, increases this fraction to $77 \%$ $(53 / 69)$, making the survey the most comprehensive in terms of redshift completeness of any sample to the full Swift depth, analyzed to date. We present the cumulative redshift distribution and derive a conservative, yet small, associated uncertainty. We constrain the fraction of Swift GRBs at high redshift to a maximum of $14 \%(5 \%)$ for $z>6(z>7)$. The mean redshift of the host sample is assessed to be $\langle z\rangle \gtrsim 2.2$, with the 10 new redshifts reducing it significantly. Using this more complete sample, we confirm previous findings that the GRB rate at high redshift $(z \gtrsim 3)$ appears to be in excess of predictions based on assumptions that it should follow conventional determinations of the star formation history of the universe, combined with an estimate of its likely metallicity dependence. This suggests that either star formation at high redshifts has been significantly underestimated, for example due to a dominant contribution from faint, undetected galaxies, or that GRB production is enhanced in the conditions of early star formation, beyond that usually ascribed to lower metallicity. Subject headings: dust, extinction — galaxies: distances and redshifts — gamma rays: bursts
\end{abstract}

\section{INTRODUCTION}

Determining the statistical properties of gamma-ray bursts (GRBs) has long been compromised by inhomogeneous selection and a bias against optically dark bursts. With Swift (Gehrels et al. 2004) it has become possible to construct much more uniform samples, and to target the host galaxies even of optically faint bursts via X-Ray Telescope (XRT) localizations, for which redshifts could not be determined from the afterglows.

We have been securing GRB host galaxy information for a homogeneous sample of 69 Swift GRBs with a large program at the Very Large Telescope (VLT). The first observations of The Optically Unbiased GRB Host (TOUGH) sample were obtained on 2006 February 23 with the survey concluding on 2008 August 29. The immediate goals are to determine the host luminosity function (LF), study the effects of reddening, determine the fraction of Ly $\alpha$ emitters in the hosts, and obtain redshifts for targets without a reported one. The sample has been carefully selected and obeys strict and welldefined criteria. To optimize the survey, we focused on systems with the best observability, which also have the

\footnotetext{
${ }^{1}$ Centre for Astrophysics and Cosmology, Science Institute, University of Iceland, Dunhagi 5, 107 Reykjavík, Iceland

2 Dark Cosmology Centre, Niels Bohr Institute, University of Copenhagen, Juliane Maries Vej 30, 2100 Copenhagen $\varnothing$, Denmark

${ }^{3}$ Centre for Astrophysics Research, University of Hertfordshire, Hatfield, Herts AL10 9AB, UK

${ }^{4}$ Department of Physics and Astronomy, University of Leicester, University Road, Leicester, LE1 7RH, UK

${ }^{5}$ Département d'Astrophysique, Géophysique et Océanographie, ULg, Allée du 6 août, 17 - Bât. B5c B4000 Liège (Sart-Tilman), Belgium
}

best available information. The survey design and catalogs are presented in Hjorth et al. (2012), the fundamental properties of the hosts in Malesani et al. (2012), the Ly $\alpha$ emission in Milvang-Jensen et al. (2012), and new VLT/X-shooter redshifts in Krühler et al. (2012).

This paper presents the first TOUGH campaign for missing redshifts. We attempted spectroscopic observations of most TOUGH host candidates with $R \lesssim 25 \mathrm{mag}$ that did not have a reported reliable redshift. A total of 19 candidates were spectroscopically observed for an aggregate of nearly $30 \mathrm{hr}$ with the aim of acquiring redshift information. In addition, we include a new redshift measurement for GRB 060908 reported in Milvang-Jensen et al. (2012, see also Fynbo et al. 2009). The details of our observations and reductions are described in the next section. New redshifts and redshift limits for each observed host are presented in $\$ 3$. We model the redshift distribution and compare it to the observed one in 4 . Finally, the implications of our observational efforts are discussed in $\$ 5$. We adopt a cosmology where the Hubble parameter is $H_{0}=70.4 \mathrm{~km} \mathrm{~s}^{-1} \mathrm{Mpc}^{-1}$, $\Omega_{\mathrm{m}}=0.27$ and $\Omega_{\Lambda}=0.73$ (Jarosik et al. 2011).

The reduced data from this work will be available from ESO6] and from the TOUGH Web site]

\section{OBSERVATIONS AND DATA REDUCTION}

The host spectroscopic observations were carried out between 2006 May 30 and 2008 August 29. The FORS2 instrument was used as well as FORS1 (Appenzeller et al. 1998) before and after the blue

\footnotetext{
6 http://archive.eso.org

7 http://www.dark-cosmology.dk/TOUGH
} 
CCD upgrade. Four different grism setups were mainly used: 300V, 300V+GG375, 600z+OG590, and $600 \mathrm{RI}+\mathrm{GG} 4358$ We requested a seeing constraint of $1^{\prime \prime} .2$ for all observations apart from GRB 060923C for which 0.8 was required (3.13). For the majority of the observations, this goal was accomplished. More detailed information is listed in Table 1 .

The data reduction was performed following standard techniques for bias and flat-field corrections. The individual spectra were then cosmic ray cleaned using the method of van Dokkum (2001). If the host trace was clearly visible in individual images, the spectra were optimally extracted for each two-dimensional image separately. Otherwise, the two-dimensional images were aligned, averaged, and the host spectrum extracted if detected.

The wavelength calibration was applied using a HeNeAr lamp spectrum obtained in the morning after the science observations. The root mean square scatter around the wavelength calibration fit was roughly $0.2 \AA$ for the $300 \mathrm{~V}$ grism and less than $0.1 \AA$ for the higher resolution grisms. The individual wavelength-calibrated spectra were averaged, and the corresponding Poisson error spectra (calculated by the IRAF/apall task) were quadratically averaged. Finally, flux calibration was applied by using standard star observations carried out every night a host was observed. We note, however, that the transparency constraint was set to "thin cirrus" implying that absolute flux calibration should be interpreted with caution.

\section{NEW REDSHIFTS AND LIMITS}

A total of 20 host systems were observed (including GRB 060908), of which only 10 had a reported optical afterglow (OA) or near-infrared afterglow (NIRA). In only two cases (GRBs 060805A and 070808) there is more than one galaxy detected within or on the border of the XRT error circle. For the other eight systems without an OA/NIRA the host identity is nearly unambiguous with the probability of chance projection being fairly low (frequently less than 5\%; Malesani et al. 2012). Below we discuss each system in detail and justify our redshift measurements and limits. When no spectral features are detected we set redshift limits in the following way:

- If the continuum is visible we estimate, using the error spectrum, the lowest wavelength at which it is significantly detected (at a significance level of around $3 \sigma$ ). The lack of $\operatorname{Ly} \alpha$ forest lines is then used to set a firm redshift upper limit.

- To get a redshift lower limit we note that GRB hosts are in general star-forming galaxies and thus display the usual emission lines, such as [O II] $\lambda 3727, \mathrm{H} \beta,[\mathrm{O}$ III] $\lambda 5007$, and $\mathrm{H} \alpha$; see e.g. the GHostS Web site 9 Table 3 in Savaglio et al. (2009), and Levesque et al. (2010). In particular, the non-detection of [O II] $\lambda 3727$ in our $300 \mathrm{~V}$ spectra immediately sets a redshift limit of $z>0.9$. This value is based on $7200 \AA$, since above it there are strong skylines which leave significant residuals in the reduced spectra.

\footnotetext{
8 See http://www.eso.org/sci/facilities/paranal/ instruments/fors/doc/ for more details.

${ }^{9}$ http://www.grbhosts.org/
}

- If neither emission lines nor a continuum are detected we follow Grupe et al. (2007) and assign a redshift upper limit to bursts with excess (above Galactic) X-ray absorbing column density 10 , calculated at $z=0$, above an equivalent hydrogen column density of $2 \times 10^{21} \mathrm{~cm}^{-2}$ (including the uncertainty) 11 Grupe et al. (2007) used an upper limit of $z=2$, but we will be slightly more conservative and assign an upper limit of $z=3.5$ to these bursts (see Equation (1) in Grupe et al. 2007). The corresponding rest-frame column density is $10^{23} \mathrm{~cm}^{-2}$. In the sample of Campana et al. (2010, see their Figure 2), no burst exceeds this number, lending support to our choice of $z \lesssim 3.5$. This approach was utilized for six events in the whole TOUGH sample. Among those, five have an $R$-band host detection, implying that their redshifts have to be $z \lesssim 6$.

\subsection{GRB 050714B (No OA/NIRA)}

There is a single faint source detected inside the XRT error circle, with a bright source close to the southern edge of the error circle. Our spectroscopic observations show the brighter one to be an M star. Neither emission lines nor a continuum are detected from the fainter object and hence no redshift information can currently be obtained for this host candidate. However, we conclude that $z \lesssim 3.5$ based on the excess column density detected in the X-ray spectrum.

\subsection{GRB 050822 (No OA/NIRA)}

In the $300 \mathrm{~V}$ spectrum, the continuum is clearly detected in the 3900-7200 $\AA$ region corresponding to the redshift range $0.9 \lesssim z \lesssim 2.2$ (left panel of Fig. 11). There is an emission line candidate $(7 \sigma)$ at $9071 \AA$ (right panel of Fig. 10 that is most likely the [O II] $\lambda 3727$ doublet at $z=1.434$. In support of this, we note that the spectral extent of the line is significantly broader than the extent of emission lines visible in other traces in the twodimensional spectrum.

At this redshift, our spectra would not $\operatorname{cover} \mathrm{H} \beta$, [O III] $\lambda 5007$, and $\mathrm{H} \alpha$. Other potential line identifications (of $9071 \AA$ ) such as $\mathrm{H} \beta$, [O III] $\lambda 5007$, and $\mathrm{H} \alpha$ are rejected on account that we would expect to see additional and roughly similarly bright lines in the wavelength range covered.

\subsection{GRB 050915A (NIRA)}

The host galaxy of the dark (Jakobsson et al. 2004) GRB 050915A was previously reported in Ovaldsen et al. (2007) and Perley et al. (2009). In 300V the continuum is clearly detected in the $4300-7200 \AA$ region without unambiguous features, corresponding to $0.9 \lesssim z \lesssim 2.5$ (Fig. 2).

In the upper panel of Fig. 2, the continuum of another brighter galaxy on the slit is visible $(z=0.444) 12$ It

10 We use the afterglow-only late spectrum (photon counting mode) obtained from http://www.swift.ac.uk/xrt_spectra/

11 For comparison, one of the sample selection criteria, as defined in Hiorth et al. (2012), is that the Galactic extinction is $A_{V} \leq$ $0.5 \mathrm{mag}$, which corresponds to a hydrogen column density of $0.9 \times$ $10^{21} \mathrm{~cm}^{-2}$ according to the relation derived by Predehl \& Schmitt (1995).

12 This value was erroneously reported as the GRB 050915A host redshift in Jakobsson et al. (2009, 2011a b c). 

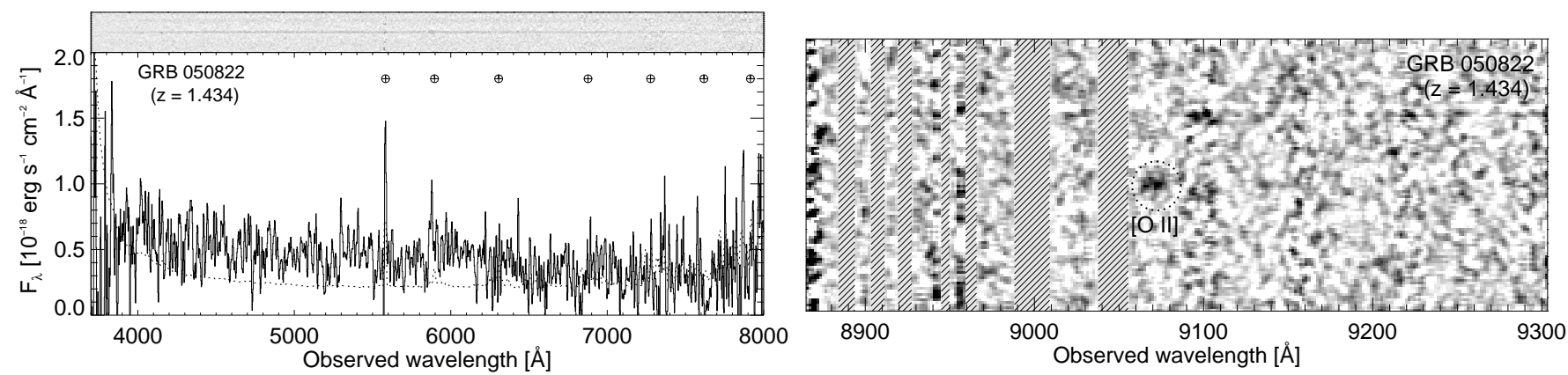

Figure 1. One- and two-dimensional spectra (left: 300V; right: 600z) of the GRB 050822 host. There are no emission lines identified in $300 \mathrm{~V}$. Telluric features and skyline residuals are marked with $\oplus$, whereas the error spectrum is plotted as a dotted line. The $600 \mathrm{z}$ emission line candidate is marked with a circle in the right panel. Hashed regions mark areas strongly affected by skylines.

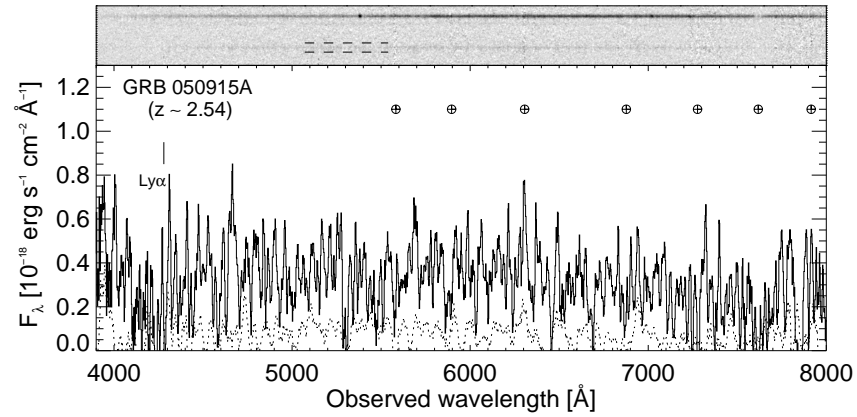

Figure 2. One- and two-dimensional spectra (300V) of the GRB 050915A host. In the upper panel, it is the bottom trace between the two horizontal dashed lines. The Ly $\alpha$ absorption feature is marked with a vertical line, whereas telluric features and skyline residuals are marked with $\oplus$. The error spectrum is plotted as a dotted line.

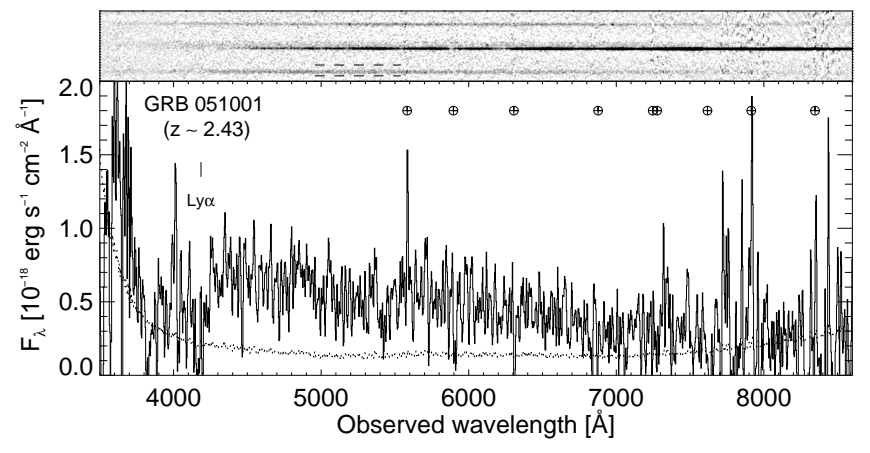

Figure 3. One- and two-dimensional spectra (300V) of the GRB 051001 host. In the upper panel, it is the bottom trace between the two horizontal dashed lines. The Ly $\alpha$ absorption feature is marked with a vertical line, whereas telluric features and skyline residuals are marked with $\oplus$. The error spectrum is plotted as a dotted line.

clearly contains flux down to around $3600 \AA$. Therefore, a spectral break must be present in the GRB 050915A host continuum, which we interpret as the $\operatorname{Ly} \alpha$ break. More specifically, the $1 \mathrm{D}$ spectrum shows a flux drop around $4300 \AA$ corresponding to $z \approx 2.54$. Recent observations by VLT/X-shooter confirm this interpretation where we detect [O II] $\lambda 3727, \mathrm{H} \beta$, and [O III] $\lambda \lambda 4959,5007$ in emission at a similar redshift (Krühler et al. 2012).

\subsection{GRB 051001 (No OA/NIRA)}

The continuum is extremely faint in the $600 \mathrm{z}$ spectrum and there are no signs of any emission lines. In 300V

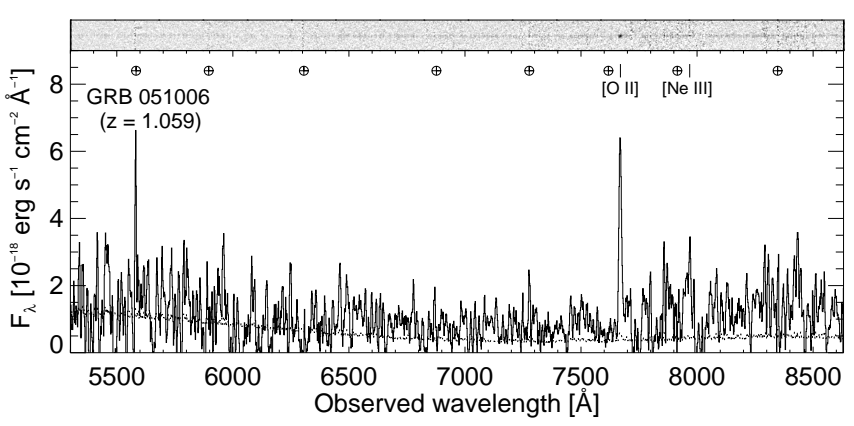

Figure 4. One- and two-dimensional spectra (600RI) of the GRB 051006 host. Emission lines are marked with vertical lines, whereas telluric features and skyline residuals are marked with $\oplus$. The error spectrum is plotted as a dotted line.

the continuum is clearly detected in the $4200-7200 \AA$ region without unambiguous features, corresponding to $0.9 \lesssim z \lesssim 2.5$ (Fig. 31). Thus, GRB 051001 can safely be ruled out as a high-redshift burst as suggested by Salvaterra et al. (2007).

The 1D spectrum shows a flux drop around $4170 \AA$ which we interpret as the Ly $\alpha$ break at $z \approx 2.43$. Recent observations by VLT/X-shooter confirm this interpretation where we detect $\mathrm{H} \beta$, [O III] $\lambda 5007$, and $\mathrm{H} \alpha$ in emission at a similar redshift (Krühler et al. 2012).

\subsection{GRB 051006 (No OA/NIRA)}

The 600RI spectrum clearly shows a strong and broad emission line which we identify as the [O II] $\lambda 3727$ doublet at $z=1.059$. At that redshift, we also detect a much weaker line, [Ne III] $\lambda 3869$ (Fig. 4). The stronger line is unlikely to be $\mathrm{H} \beta$, [O III] $\lambda 5007$, or $\mathrm{H} \alpha$ since additional and roughly similarly bright lines would be expected in the wavelength range covered.

\subsection{GRB 051117B (No OA/NIRA)}

Our $300 \mathrm{~V}$ spectrum clearly shows a strong emission line in addition to a few absorption features (Fig. 5). These features are consistent with being [O II] $\lambda 3727$, $\mathrm{H} 10, \mathrm{H} 9, \mathrm{H} 8, \mathrm{H} \varepsilon$ and $\mathrm{Ca}$ II $\mathrm{K}$ and $\mathrm{H}$ at a common redshift of $z=0.481$. We note that $\mathrm{H} \varepsilon$ and $\mathrm{Ca}$ II $\mathrm{H}$ are blended with a strong skyline residual.

\subsection{GRB 060306 (No OA/NIRA)}

Neither emission lines nor a continuum were detected in the $600 \mathrm{z}$ spectrum and hence no redshift information could be obtained. However, there is a faint detection of 


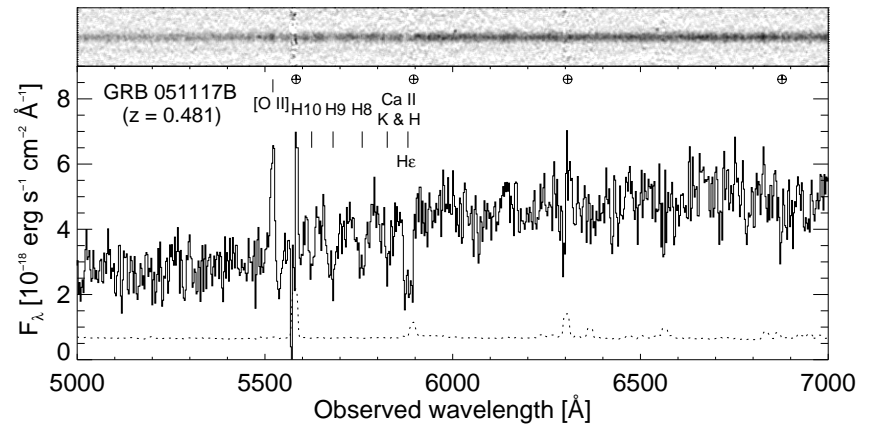

Figure 5. One- and two-dimensional spectra (300V) of the GRB 051117B host. Line features are marked with vertical lines, whereas telluric features and skyline residuals are marked with $\oplus$. The error spectrum is plotted as a dotted line.

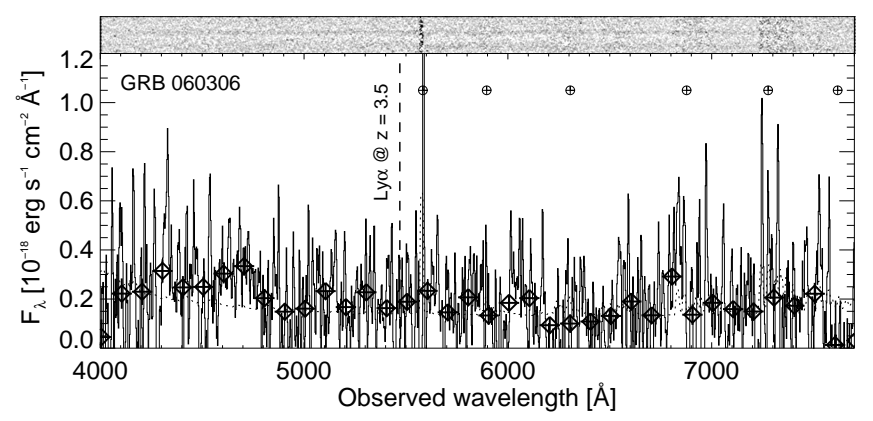

Figure 6. One- and two-dimensional spectra (300V) of the GRB 060306 host. Telluric features and skyline residuals are marked with $\oplus$, whereas the error spectrum is plotted as a dotted line. The diamonds show the rebinned (100 $\AA)$ spectrum. The vertical dashed line indicates the location of Ly $\alpha$ if $z=3.5$ (Salvaterra et al. 2012)

the continuum in the $300 \mathrm{~V}$ spectrum in the approximate wavelength range 4300-6800 A (Fig. 6) indicating $0.8 \lesssim$ $z \lesssim 2.5$.

In particular, as apparent from the rebinned spectrum (diamonds in Fig. 6), we do not detect any flux break corresponding to Ly $\alpha$ at $z=3.5$. This redshift was based on the detection of a single emission line interpreted as [O II] (Salvaterra et al. 2012). We note that this lack of a break is different from what we detect from $z>2$ hosts presented in this paper (GRBs 050915A, 051001, and 070129). We tentatively suggest that the redshift must be either $z=2.35$ or $z=1.55$ if the emission line is interpreted as [O III] or $\mathrm{H} \alpha$, respectively.

\subsection{GRB 060719 (NIRA)}

The continuum is very faint in $600 \mathrm{z}$ but slightly brighter in $300 \mathrm{~V}$ (Fig. 77). Based on the continuum in the $3600-7200 \AA$ region we infer $0.9 \lesssim z \lesssim 2.0$. Indeed, recent VLT/X-shooter observations have detected emission lines (strong $\mathrm{H} \alpha$ and an indication of [O II] $\lambda 3727$ ) outside of our grism wavelength range, consistent with the FORS redshift limit (Krühler et al. 2012).

\subsection{GRB 060805A (No OA/NIRA)}

There are two host galaxy candidates within the XRT error circle, a bright object (A) at the southwestern edge and a second, fainter source (B) slightly northeast of the center (Perley et al. 2009; Malesani et al. 2012). Our slit covered both objects. No line features are observed over

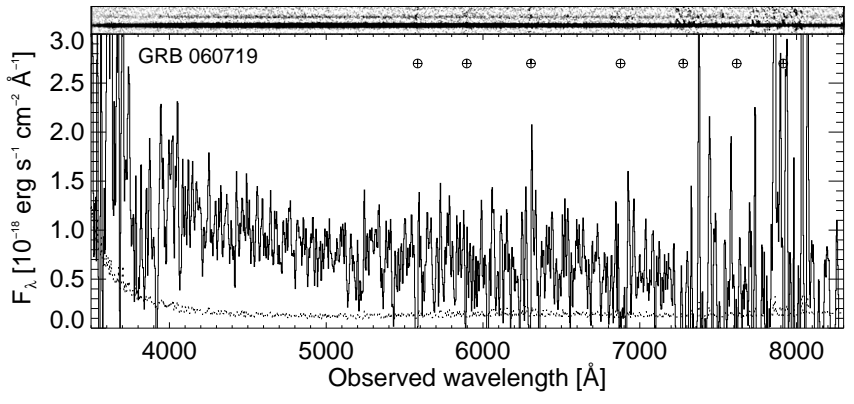

Figure 7. One- and two-dimensional spectra (300V) of the GRB 060719 host. In the upper panel, it is the fainter upper trace. Telluric features and skyline residuals are marked with $\oplus$, whereas the error spectrum is plotted as a dotted line.

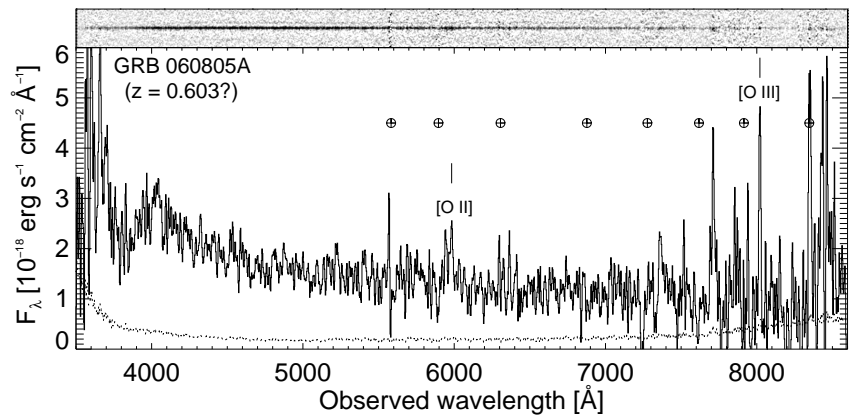

Figure 8. One- and two-dimensional spectra (300V) of the brighter galaxy (object A) within the XRT error circle of GRB 060805A. The fainter galaxy (object B) is barely seen in the two-dimensional spectrum just above object $\mathrm{A}$. Line features are marked with vertical lines, whereas telluric features and skyline residuals are marked with $\oplus$. The error spectrum is plotted as a dotted line.

the spectral range for object $B$. There is, however, a faint continuum detectable by block averaging the spectrum along the dispersion axis. It is detected down to approximately $4200 \AA$ which corresponds to $z \lesssim 2.5$.

The continuum of object $\mathrm{A}$ is detected down to at least $3600 \AA$ which implies $z \lesssim 2.0$ (Fig. 8), consistent with the findings of Perley et al. (2009). The spectrum also displays [O II] $\lambda 3727$ and [O III] $\lambda 5007$ at a common redshift of $z=0.603$. The probability of chance projection for objects $\mathrm{A}$ and $\mathrm{B}$ is $1.6 \%$ and $6.6 \%$, respectively, calculated following the prescription in Bloom et al. (2002). Hence, the host identification is ambiguous, and we cannot claim to have secured the redshift of this burst.

\subsection{GRB 060814 (NIRA)}

The host system of GRB 060814 seems to consist of three different blobs (Malesani 2006) which we mark as A, B, and C (Fig. 9). The location of the NIRA (Levan et al. 2006) is consistent with blob A; we used the data from Levan et al. (2006) along with our images to precisely position the afterglow on the host complex. Thöne et al. (2007) reported a redshift of $z=0.84$ although it is not clear which part of the complex was covered by their slit.

Our spectra clearly show emission lines emanating from the combined region of blobs B and C (Fig. 10]). In the $600 \mathrm{z}$ spectrum (seeing: $1^{\prime \prime} .3$ ), we detect $\mathrm{H} \beta$ and [O III] $\lambda \lambda 4959,5007$ at a common redshift of $z=0.841$. There is an indication of [O II] $\lambda 3727$ in the $300 \mathrm{~V}$ spec- 


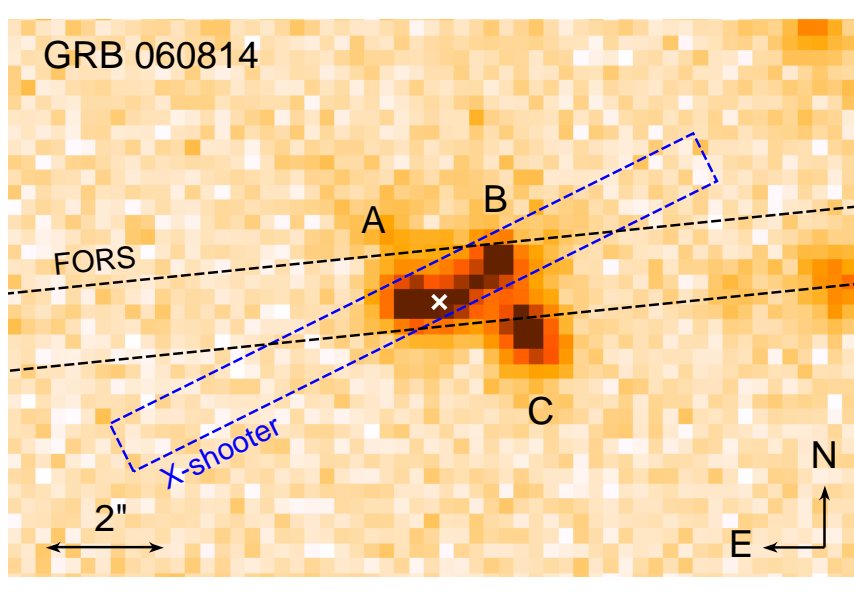

Figure 9. $R$-band image (seeing: $0 . ' 65$ ) of the GRB 060814 field. The location of the NIR afterglow is marked with a cross and is consistent with blob A. The orientation and extent of the FORS slit is also shown; it mostly cover blobs A and B, and partly blob C. The X-shooter slit only covers blobs A and B.

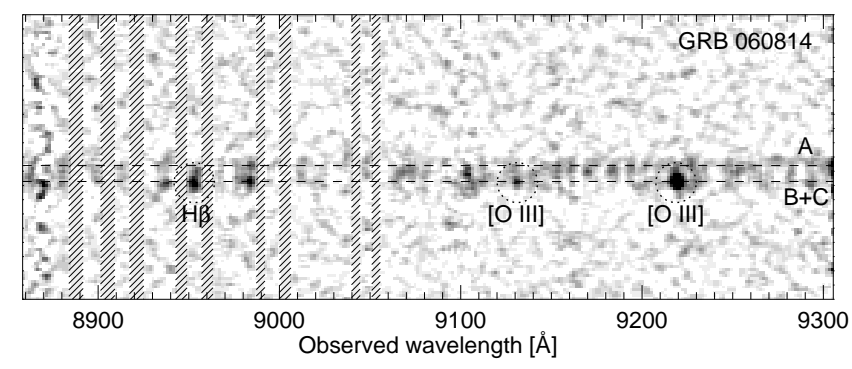

Figure 10. Two-dimensional spectrum (600z) of the GRB 060814 host complex. The dashed upper (lower) line indicates the centroid of blob A $(\mathrm{B}+\mathrm{C})$ which is shown in Fig. 9 Hashed regions mark areas strongly affected by skylines.

trum but residuals from strong sky lines make the identification ambiguous with our resolution.

However, there are no indications of any emission lines at the location of blob A. A clear detection of the continuum in the $300 \mathrm{~V}$ spectrum in the approximate range $3600-7200 \AA$ suggests that $0.9 \lesssim z \lesssim 2.0$. In any case, the conclusion is that a host redshift of $z=0.84$ is not supported by our data. Indeed, recent VLT/Xshooter observations led to the detection of [O II] $\lambda 3727$, [O III] $\lambda \lambda 4959,5007$, and $\mathrm{H} \alpha$ in emission at $z=1.92$ (Krühler et al. 2012), clearly visible in blobs A and B (the slit did not cover blob $\mathrm{C}$ as shown in Fig. 9) [13 At this redshift, the most prominent emission lines are outside of our FORS grisms wavelength range. The necessary consequence of these observations taken together is that the host complex consists of blobs $\mathrm{A}$ and $\mathrm{B}$ at $z=1.92$, while blob $\mathrm{C}$ is a chance alignment at $z=0.84$.

\subsection{GRB $060908(O A)$}

A Ly $\alpha$ emission line is detected from the host galaxy at a redshift of $z=1.884$ (part of the TOUGH Ly $\alpha$ campaign: Milvang-Jensen et al. 2012). Fynbo et al. (2009) utilized these findings and confirmed the redshift by detecting the C IV $\lambda \lambda 1548,1550$ doublet in the OA spectrum, ruling out the tentative redshift of $z=2.43$ re-

13 Our analysis is consistent with what is reported by Salvaterra et al. (2012) who used the same dataset.

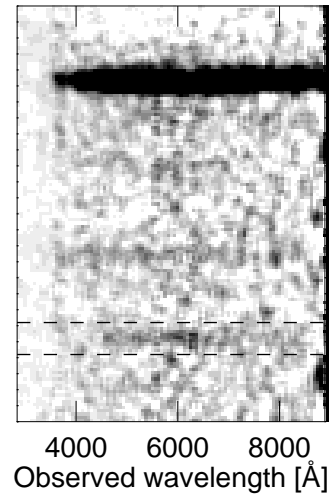

Figure 11. Two-dimensional spectrum (300V) of the GRB 060923A host. It has been block averaged along the dispersion axis to make the host continuum visible. It is the bottom trace between the two horizontal dashed lines.

ported by Rol et al. (2006).

\subsection{GRB 060923A (NIRA)}

The extremely red afterglow of GRB 060923A was discussed in Tanvir et al. (2008). Their later-time optical imaging and spectroscopy revealed a faint galaxy coincident with the GRB position which implied a moderate redshift and therefore that dust is the likely cause of the very red afterglow color.

Our $300 \mathrm{~V}$ spectrum reveals a very faint continuum with a possible weak emission line candidate at around $6330 \AA$ ( $3 \sigma$ detection). In order to verify this we obtained a higher resolution 1200R spectrum. There is again a weak detection of the continuum but no sign of an emission line at the aforementioned wavelength. In Fig. 11 the continuum is detected down to approximately $4600 \AA$ which corresponds to $z \lesssim 2.8$ (Tanvir et al. 2008).

\subsection{GRB 060923C (NIRA)}

The GRB 060923C NIRA was located only around 1.5 away from an $R=20.9 \mathrm{mag}$ bright star. Detecting the host ( $R=25.5 \mathrm{mag}$ ) spectrum, even under good seeing conditions, is therefore quite a challenge. As can be seen in the upper panel of Fig. 12, the stellar spectrum overwhelms any signs of the host continuum. However, there is an indication of an emission line at the expected location of the host trace at approximately $6942 \AA$. In order to get a better view of the situation we subtracted the star continuum using a two-dimensional deconvolution technique.

The deconvolution method applied to the spectrum is presented in detail in Courbin et al. (2000), resulting in a spatially deconvolved spectrum with the stellar spectrum separated from the spectrum of the host galaxy. This spatial separation of the pointlike component from the diffuse one relies on an accurate building of the pointspread function and on the hypothesis that the host contains no significant structure narrower than the fixed and finite resolution of the deconvolved spectrum. Following Letawe et al. (2008), we have chosen to subtract the stellar spectrum, as derived from the deconvolution process, from the original spectrum. This avoids smoothing of the diffuse component mandatory for a proper separation between the star and host. Thus, we obtain a two-dimensional host galaxy spectrum with a spatial res- 

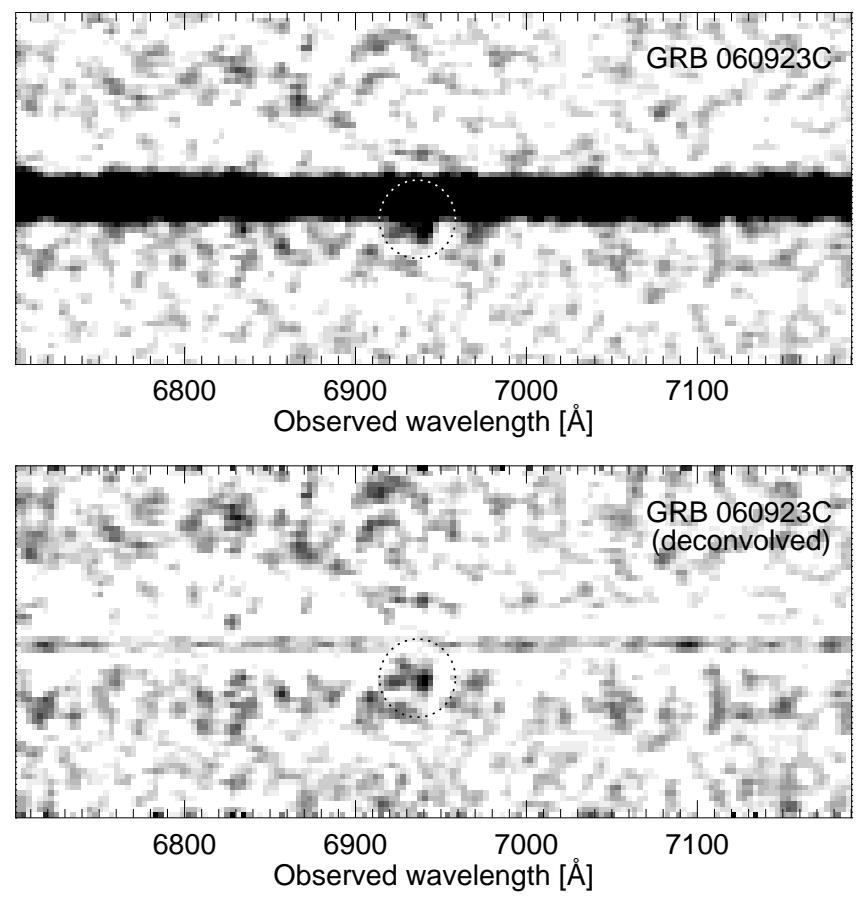

Figure 12. Original (upper panel) and deconvolved (lower panel) two-dimensional spectrum (300V) of the GRB 060923C host. The emission line candidate is indicated with a circle.

olution constant along the slit and equal to that of the original data

The results are shown in the lower panel of Fig. 12 The emission line candidate is still visible. If real, the identification could only be with Ly $\alpha$ or $\left[\begin{array}{ll}\mathrm{O} & \mathrm{II}\end{array}\right] 3727$ in which case the redshift would be $z=4.71$ or $z=$ 0.86 , respectively. We have considered other potential line identifications such as $\mathrm{H} \beta$, [O III] $\lambda 5007$, or $\mathrm{H} \alpha$ all of which are rejected on account that we would expect to see additional and roughly similarly bright lines in the wavelength range covered. We note that the Grupe et al. (2007) method implies that $z \lesssim 3.5$ so that $z=4.71$ is excluded.

\subsection{GRB 061021 (OA)}

Our 300V host spectrum shows [O II] $\lambda 3727$ and [O III] $\lambda \lambda 4959,5007$ at a common redshift of $z=0.345$ (Fig. 13). Using this redshift information, Fynbo et al. (2009) identified the $\mathrm{Mg}$ II $\lambda \lambda 2796,2803$ doublet in the very blue end of the afterglow spectrum.

\subsection{GRB 070103 (No OA/NIRA)}

The host continuum detection down to $7500 \AA$ in our $600 \mathrm{z}$ spectrum only allows us to set a modest limit of $z \lesssim 5.2$ (Fig. 14). This is less constraining than using the Grupe et al. (2007) method which implies that $z \lesssim 3.5$ (Fynbo et al. 2009). There are no convincing emission lines present. However, recent VLT/X-shooter observations have detected [O III] $\lambda \lambda 4959,5007$ in emission outside of our grism wavelength range, consistent with the aforementioned redshift limits (Krühler et al. 2012).

\subsection{GRB 070129 (OA)}

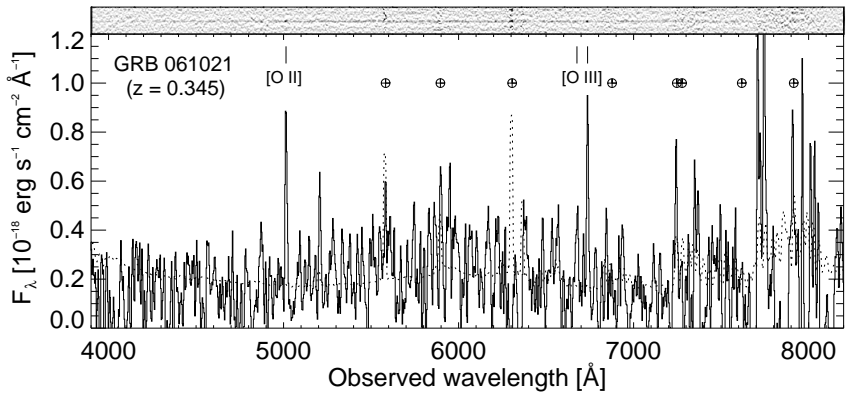

Figure 13. One- and two-dimensional spectra (300V) of the GRB 061021 host. Emission lines are marked with vertical lines, whereas telluric features and skyline residuals are marked with $\oplus$. The error spectrum is plotted as a dotted line.

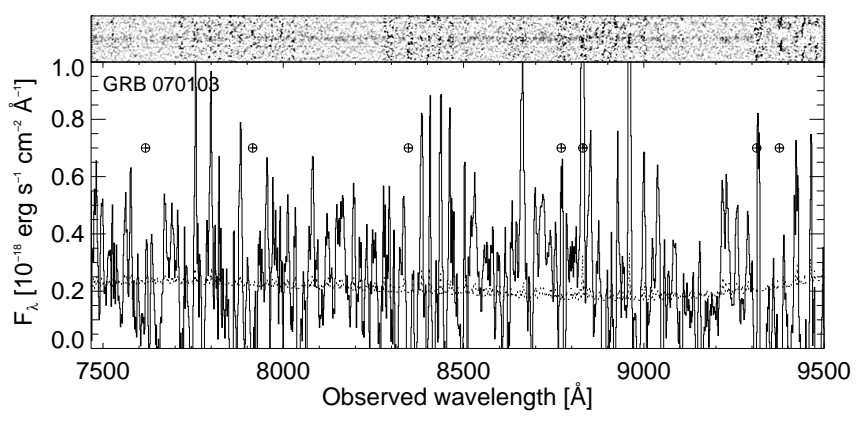

Figure 14. One- and two-dimensional spectra $(600 \mathrm{z})$ of the GRB 070103 host. Telluric features and skyline residuals are marked with $\oplus$, whereas the error spectrum is plotted as a dotted line.

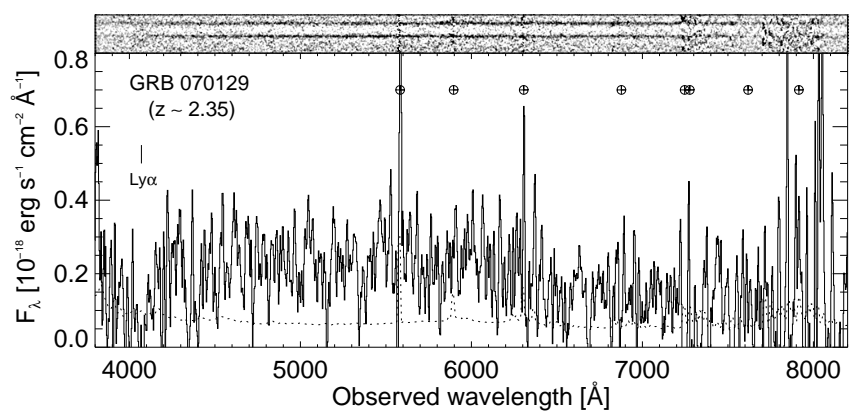

Figure 15. One- and two-dimensional spectra (300V) of the GRB 070129 host (lower trace). The Ly $\alpha$ absorption feature is marked with a vertical line, whereas telluric features and skyline residuals are marked with $\oplus$. The error spectrum is plotted as a dotted line.

Neither emission lines nor a continuum were detected in the $600 \mathrm{z}$ spectrum and hence no redshift information could be obtained. However, there is a clear detection of the continuum in the $300 \mathrm{~V}$ spectrum between approximately 4100 and $7200 \AA$, suggesting that $0.9 \lesssim z \lesssim 2.4$. Thus, we can immediately rule GRB 070129 out as a high-redshift candidate as suggested by Salvaterra et al. (2007).

In the upper panel of Fig. 15, the continuum of another similarly bright object on the slit is visible. It clearly contains flux down to around $3900 \AA$. Therefore, a spectral break is present in the GRB 070129 host continuum, which we interpret as the Ly $\alpha$ break. More specifically, the $1 \mathrm{D}$ spectrum shows a flux drop around $4070 \AA$ corresponding to $z \approx 2.35$. Recent observations by VLT/X- 


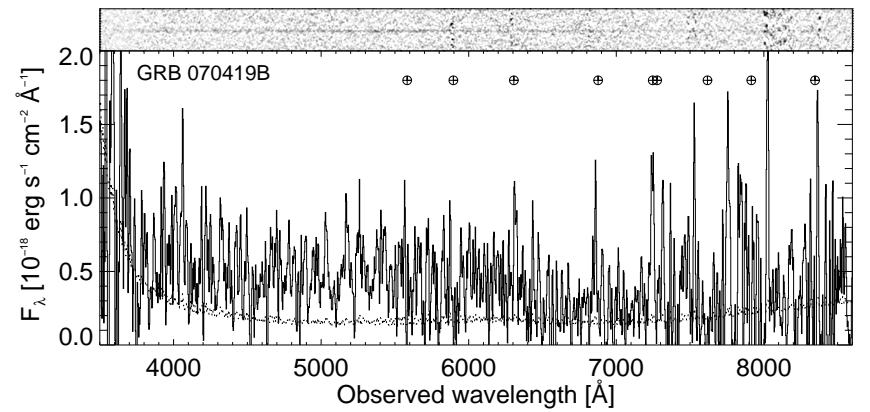

Figure 16. One- and two-dimensional spectra (300V) of the GRB 070419B host. Telluric features and skyline residuals are marked with $\oplus$, whereas the error spectrum is plotted as a dotted line.

shooter confirm this interpretation where we detect [O II] $\lambda 3727$, [O III] $\lambda \lambda 4959,5007$, and $\mathrm{H} \alpha$ in emission at a similar redshift (Krühler et al. 2012).

\subsection{GRB 070306 (NIRA)}

The highly extinguished afterglow of GRB 070306 and the properties of its host galaxy were discussed in Jaunsen et al. (2008). The OA low-resolution spectrum displayed a single emission line which was tentatively interpreted as [O II] $\lambda 3727$. In an attempt to verify this interpretation by resolving the doublet we observed the host galaxy with the higher-resolution $1028 \mathrm{z}$ grism as part of TOUGH. As shown in Figure 4 in Jaunsen et al. (2008) the doublet was successfully resolved and a redshift of $z=1.496$ confirmed.

\subsection{GRB 070328 (No OA/NIRA)}

A very faint host continuum detection down to $7500 \AA$ in our $600 \mathrm{z}$ spectrum only allows us to set a modest limit of $z \lesssim 5.2$. This is less constraining than using the Grupe et al. (2007) method which implies that $z \lesssim 3.5$ (Fynbo et al. 2009). There are no convincing emission lines present.

\subsection{GRB 070419B (OA)}

The continuum is detected in the $300 \mathrm{~V}$ spectrum but without any significant emission lines visible (Fig. 16). There is an unambiguous detection of the continuum between approximately 3900 and $7200 \AA$ indicating that $0.9 \lesssim z \lesssim 2.2$. Indeed, recent VLT/X-shooter observations have detected [O III] $\lambda \lambda 4959,5007$ and $\mathrm{H} \alpha$ in emission outside of our grism wavelength range, consistent with the FORS redshift limit (Krühler et al.|2012).

\subsection{GRB 070808 (No OA/NIRA)}

There is a faint $(R=26.7)$ object consistent with the $\mathrm{X}$-ray position, as well as two brighter ones just outside the X-ray error circle. We obtained a 600RI spectrum of the brightest source (object A: Malesani et al. 2012). It clearly shows two emission lines which we identify as the [O II] $\lambda 3727$ doublet and $\mathrm{H} \beta$ at a common redshift of $z=0.681$ (Fig. 17). The probability of chance projection for this object is around $4 \%-5 \%$, calculated following the prescription in Bloom et al. (2002), moreover it definitely lies outside the UVOT-enhanced XRT error circle. The fainter object is consistent with the XRT error circle, but it also has a large chance superposition probability

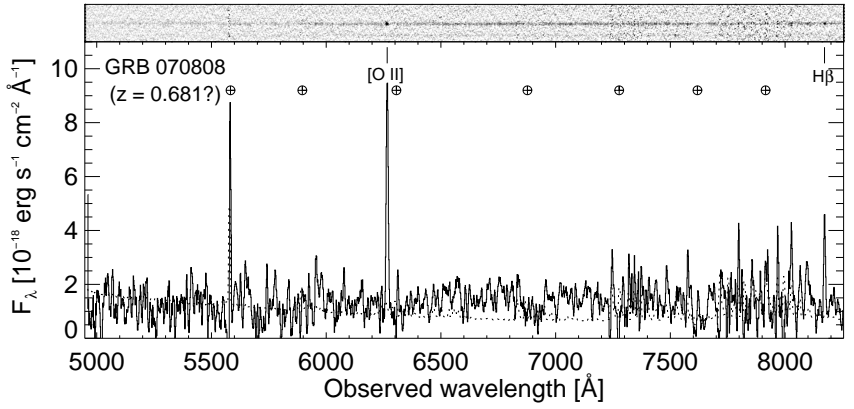

Figure 17. One- and two-dimensional spectra (600RI) of object A located close to the NE border of the GRB 070808 XRT error circle. Emission lines are marked with vertical lines, whereas telluric features and skyline residuals are marked with $\oplus$. The error spectrum is plotted as a dotted line.

(25\%). Hence, we cannot claim to have secured the redshift of this burst. However, we conclude that $z \lesssim 3.5$ based on the excess column density detected in the $\widetilde{\mathrm{X}}$-ray spectrum.

\subsection{Summary}

We report the redshifts of 10 GRB host galaxies whose average redshift is $\langle z\rangle=1.59$, significantly lower than the overall Swift GRB mean redshift. The low value is most likely the result of targeting the brightest galaxies in the sample $(R \lesssim 25 \mathrm{mag})$. GRB 061021 is among the closest "classical" Swift long-duration burst detected so far. Furthermore, we have estimated redshift limits for an additional five hosts and inferred that three burst redshifts reported in the literature are erroneous (GRBs 060306, 060814, and 060908). The results are listed in Table 1 along with the observational details. We have also estimated the emission line flux and the equivalent width (EW) of most line features detected and detailed them in Table 2

\section{THE GRB REDSHIFT DISTRIBUTION}

\subsection{Redshift Measurements and Constraints}

Figure 18 shows the cumulative redshift distribution of the 53 TOUGH bursts with a measured redshift, including four photometric redshifts and the new VLT/Xshooter redshifts presented in Krühler et al. (2012) (see Table 3 in Hjorth et al. 2012). Also plotted is a conservative systematic error band (hatched region) containing information for all the 69 TOUGH bursts. The shaded region represents the likely statistical ( $1 \sigma$ standard error of the sample) uncertainty of the measured redshift distribution under the assumption that it is a true random sample of the overall population.

The hatched region incorporates the information presented in Table 1, including the redshift limits. In addition, the upper boundary is produced by simply placing GRBs with only upper limits to their redshifts at $z=0$. The lower boundary is generated in the following way. GRBs with a detected OA and/or host are placed at the maximum redshift they can have, given their bluest photometric detection reported in the literature or in Hiorth et al. (2012). Following the general decline of the Ly $\alpha$ forest density with decreasing redshift (e.g. Songaila 2004), we set two different limits depending on the wavelength region probed by the available 
Table 1

New Redshifts and Redshift Limits Determined in the Host Galaxy Sample

\begin{tabular}{|c|c|c|c|c|c|c|c|c|c|}
\hline$\overline{\text { GRB }}$ & OA or NIRA? & Redshift & $\begin{array}{l}R_{\text {host }} \\
\text { (mag) }\end{array}$ & Instrument & Grism+Filter & $\begin{array}{l}\text { Slit Width } \\
\left({ }^{\prime \prime}\right)\end{array}$ & $\begin{array}{l}\text { Seeing } \\
\left({ }^{\prime \prime}\right)\end{array}$ & $\begin{array}{c}\text { Spectral Res. } \\
(\AA)\end{array}$ & $\begin{array}{l}\text { Exp. Time } \\
(\mathrm{s})\end{array}$ \\
\hline \multirow{2}{*}{ 050714B } & \multirow{2}{*}{ No } & \multirow{2}{*}{$z \lesssim 3.5$} & \multirow{2}{*}{25.5} & oFORS1 & $300 \mathrm{~V}$ & 1.3 & 0.9 & 11.9 & $4 \times 1220$ \\
\hline & & & & nFORS1 & $300 \mathrm{~V}$ & 1.3 & 0.7 & 7.3 & $4 \times 1220$ \\
\hline \multirow{2}{*}{050822} & \multirow{2}{*}{ No } & \multirow{2}{*}{1.434} & \multirow{2}{*}{24.2} & oFORS1 & $300 \mathrm{~V}+\mathrm{GG} 375$ & 1.3 & 0.8 & 10.6 & $2 \times 1300$ \\
\hline & & & & FORS2 & $600 z+O G 590$ & 1.0 & 1.9 & 3.2 & $2 \times 1275$ \\
\hline 050915A & NIRA & $z \approx 2.54$ & 24.6 & oFORS1 & $300 \mathrm{~V}$ & 1.3 & 1.1 & 14.5 & $4 \times 1295$ \\
\hline \multirow{2}{*}{051001} & \multirow{2}{*}{ No } & \multirow{2}{*}{$z \approx 2.43$} & \multirow{2}{*}{24.4} & nFORS1 & $300 \mathrm{~V}$ & 1.3 & 0.8 & 8.4 & $4 \times 1360$ \\
\hline & & & & FORS2 & $600 z+O G 590$ & 1.0 & 0.7 & 2.3 & $2 \times 1350$ \\
\hline \multirow{2}{*}{$\begin{array}{l}051006 \\
051117 \mathrm{~B}\end{array}$} & No & \multirow{2}{*}{$\begin{array}{l}1.059 \\
0.481\end{array}$} & \multirow{2}{*}{$\begin{array}{l}23.0 \\
21.1\end{array}$} & FORS2 & 600RI+GG435 & 1.0 & 1.3 & 3.3 & $2 \times 430$ \\
\hline & No & & & oFORS1 & $300 \mathrm{~V}$ & 1.3 & 1.3 & 17.2 & $1 \times 1000$ \\
\hline \multirow{2}{*}{060306} & \multirow{2}{*}{ No } & \multirow{2}{*}{$0.8 \lesssim z \lesssim 2.5$} & \multirow{2}{*}{24.1} & oFORS1 & $300 \mathrm{~V}+\mathrm{GG} 375$ & 1.3 & 1.3 & 17.2 & $2 \times 1300$ \\
\hline & & & & FORS2 & $600 \mathrm{z}+\mathrm{OG} 590$ & 1.0 & 1.1 & 3.2 & $2 \times 1275$ \\
\hline \multirow{2}{*}{060719} & \multirow{2}{*}{ NIRA } & \multirow{2}{*}{$0.9 \lesssim z \lesssim 2.0$} & \multirow{2}{*}{24.6} & nFORS1 & $300 \mathrm{~V}$ & 1.3 & 0.9 & 9.4 & $4 \times 1360$ \\
\hline & & & & FORS2 & $600 z+O G 590$ & 1.0 & 1.0 & 3.2 & $4 \times 1350$ \\
\hline \multirow{2}{*}{ 060805A } & \multirow{2}{*}{ No } & \multirow{2}{*}{$z \lesssim 2.5$} & \multirow{2}{*}{$\begin{array}{l}23.5(\mathrm{~A}) \\
25.1(\mathrm{~B})\end{array}$} & nFORS1 & $300 \mathrm{~V}$ & 1.3 & 1.3 & 13.6 & $4 \times 1295$ \\
\hline & & & & FORS2 & $600 z+O G 590$ & 1.0 & 1.4 & 3.2 & $1 \times 1500$ \\
\hline \multirow{2}{*}{060814} & \multirow{2}{*}{ NIRA } & 1.92 & 22.9 & nFORS1 & $300 \mathrm{~V}$ & 1.3 & 0.8 & 8.4 & $2 \times 1295$ \\
\hline & & & & FORS2 & $600 z+O G 590$ & 1.0 & 1.3 & 3.2 & $2 \times 1350$ \\
\hline 060908 & OA & 1.884 & 25.5 & nFORS1 & $600 \mathrm{~B}$ & 1.3 & 0.8 & 6.5 & $4 \times 1345$ \\
\hline 060923A & NIRA & $z<2.8$ & 26.1 & nFORS1 & $300 \mathrm{~V}$ & 1.3 & 1.0 & 10.5 & $2 \times 1280$ \\
\hline & NHed & & & FORS2 & $1200 \mathrm{R}+\mathrm{GG} 435$ & 1.3 & 1.0 & 1.5 & $2 \times 1350$ \\
\hline 060923C & NIRA & $z \lesssim 3.5$ & 25.5 & nFORS1 & $300 \mathrm{~V}$ & 1.0 & 0.8 & 8.4 & $4 \times 1360$ \\
\hline 061021 & OA & 0.345 & 24.4 & nFORS1 & $300 \mathrm{~V}$ & 1.3 & 0.7 & 7.3 & $2 \times 1280$ \\
\hline 070103 & No & $z \lesssim 3.5$ & 24.2 & FORS2 & $600 z+O G 590$ & 1.0 & 1.0 & 3.2 & $4 \times 900$ \\
\hline 070129 & OA & $z \approx 2.35$ & 24.4 & nFORS1 & $300 \mathrm{~V}$ & 1.3 & 1.4 & 13.6 & $4 \times 1360$ \\
\hline & & & & FORS2 & $600 z+O G 590$ & 1.0 & 1.4 & 3.2 & $2 \times 1275$ \\
\hline 070306 & NIRA & 1.496 & 22.9 & FORS2 & $1028 \mathrm{z}+\mathrm{OG} 590$ & 1.0 & 0.7 & 2.5 & $2 \times 1300$ \\
\hline 070328 & No & $z \lesssim 3.5$ & 24.4 & FORS2 & $600 z+O G 590$ & 1.0 & 1.0 & 3.2 & $5 \times 1330$ \\
\hline 070419B & OA & $0.9 \lesssim z \lesssim 2.2$ & 25.2 & nFORS1 & $300 \mathrm{~V}$ & 1.3 & 0.9 & 9.4 & $6 \times 1360$ \\
\hline 070808 & No & $z \lesssim 3.5$ & 26.7 & FORS2 & 600RI+GG435 & 1.3 & 2.2 & 4.3 & $1 \times 715$ \\
\hline
\end{tabular}

Note. $-R_{\text {host }}$ is the $R$-band total magnitude (or $3 \sigma$ upper limit) of the host galaxy (before correcting for Galactic extinction) from Malesani et al. (2012). The GRB 060805A host redshift limit is conservative and refers to the fainter object (B) within the XRT error circle. The GRB 060908 host redshift originates from the Ly $\alpha$ part of TOUGH (Milvang-Jensen et al. 2012). The host redshifts of GRBs 060719, 070103, and 070419B are reported in Krühler et al. (2012) and stem from our VLT/X-shooter observations. The redshift uncertainty is of the order of \pm 0.001 for those given to three decimal places. Data obtained with FORS1 before 2007 April 6 are marked oFORS1. Data obtained after the FORS1 blue CCD upgrade are labeled nFORS1. The wavelength coverage for each setup is approximately $3500-8140 \AA$ (oFORS1/300V/ $1{ }^{\prime \prime}$ ) 3 ), 3500-9640 $\AA$ (nFORS1/300V/1.' 0), 3500-8880 $\AA$ (nFORS1/300V/ $1^{\prime \prime} 3$ ), $7470-$

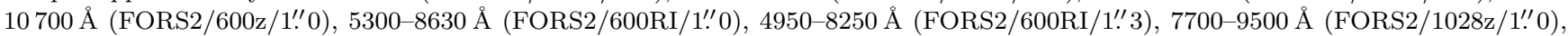
and $5720-7200 \AA$ (FORS2/1200R/1". 3).

observations. At low redshift, the opacity of the $\mathrm{Ly} \alpha$ forest is small, hence, for detections in blue and optical filters ( $V$ band and blueward), the maximum redshift is derived by comparing the reddest wavelength in the filter response curve to the Lyman limit. At higher redshift, only negligible flux survives blueward of the Ly $\alpha$ wavelength, therefore, for redder filters, the comparison is to Ly $\alpha$ (e.g. Dall'Aglio et al. 2008). If a more constraining limit is not available, we can set their maximum redshift to $z=3.5$ if their X-ray spectra fulfill the criterion described in 3 . For only a single burst, GRB 061004, are there no redshift constraints available. It is arbitrarily placed at a maximum redshift of $z=10$ in Fig. 18 .

\subsection{Modelling}

Various authors have attempted to predict or model the GRB redshift distribution and hence compare it to observations (e.g. Gorosabel et al. 2004; Natarajan et al. 2005; Jakobsson et al. 2006; Le \& Dermer 2007; Salvaterra \& Chincarini 2007; Guetta \& Piran 2007; Tanvir \& Jakobsson 2007; Kistler et al. 2008; Dong \& Lu 2009; Campisi et al. 2010; Robertson \& Ellis 2012), but of necessity have worked with samples with considerably greater incompleteness and optical bias than TOUGH now provides.
During the review process of this work, Salvaterra et al. (2012) presented the redshift distribution of a complete sample of Swift GRBs, which was inspired by criteria similar to those applied to TOUGH. Their sample is slightly smaller and, by construction, limited to gamma-ray bright events. The TOUGH sample extends to fainter luminosities (a factor of around six) and as such is more suited to test the faint (and potentially high-redshift) end of the GRB distribution. Indeed, their mean (median) redshift is 1.84 (1.64), significantly lower than for the whole TOUGH sample.

Given the association of long GRBs with the deaths of massive stars (e.g. Hjorth et al. 2003; Wooslev \& Bloom 2006), it is commonly assumed that the GRB rate density follows the star-formation rate (SFR) density history (e.g. Paczvński 1998; Wijers et al. 1998; Porciani \& Madau 2001; Jakobsson et al. 2005; Fynbo et al. 2008), with a possible low-metallicity enhancement (e.g. Langer \& Norman 2006; Li 2008; Butler et al. 2010) and/or evolution with redshift in intrinsic rate or LF (e.g. Coward 2005; Daigne et al. 2006; Guetta \& Piran 2007; Kistler et al. 2008; Salvaterra et al. 2009). For the purposes of this observational paper, we will only present illustrative model fits. 


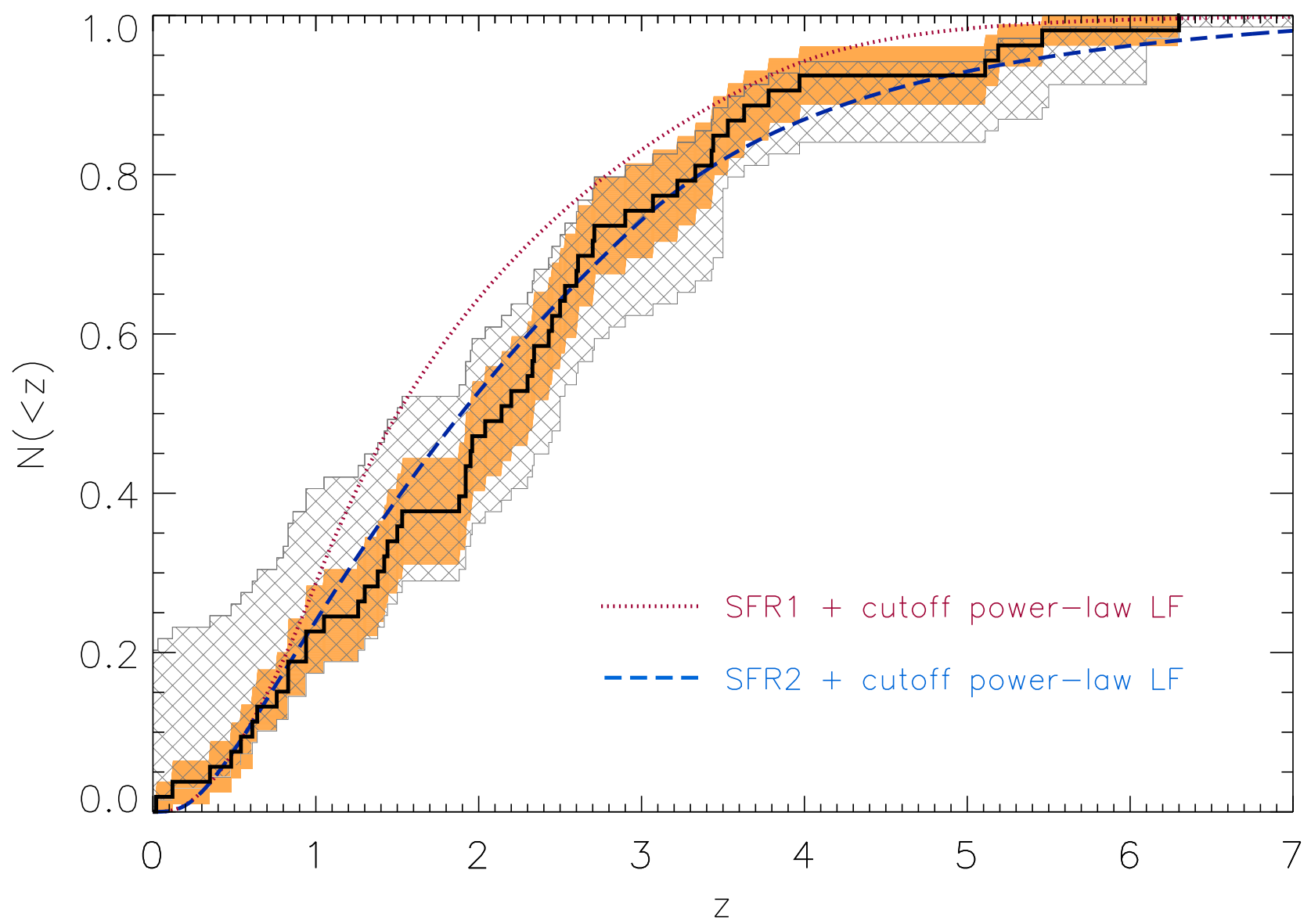

Figure 18. Thick solid curve: the cumulative fraction of GRBs as a function of redshift for the 53 Swift bursts in the TOUGH sample with a measured redshift $(\langle z\rangle=2.23)$. Hatched region: this is a conservative error region showing the systematic error on the thick solid curve. Shaded region: statistical region showing the $1 \sigma$ sampling error band around the thick solid curve. Dotted curve: the expected redshift distribution for Swift observable long GRBs using the SFR1 history parameterization (see the main text). Dashed curve: the same redshift distribution for the SFR2 history parameterization (see the main text).

Currently, we have little guidance from theory as to plausible functional forms for the GRB LF, and various ones have been considered in the above studies. Two of the most commonly adopted forms are either a broken power law (e.g. Guetta et al. 2005; Guetta \& Piran 2007; Butler et al. 2010; Wanderman \& Piran 2010), or a single power law with an exponential cutoff at low luminosities (e.g. Natarajan et al. 2005; Campisi et al. 2010; Cao et al. 2011). Both forms generally underpredict the rate of low-luminosity GRBs (as exemplified by GRB 980425), suggesting that such bursts may form a separate population (Cobb et al. 2006: Soderberg et al. 2006; Liang et al. 2007; Coward et al. 2008; Zitouni et al. 2008; Foley et al. 2008; Virgili et al. 2009). In our homogeneous and unbiased TOUGH sample, only one event (GRB 060218) is generally regarded as a member of this faint population, though we note this represents a rate of nearby bursts consistent with that predicted from the BATSE sample by Chapman et al. (2007). For the sake of clarity and simplicity, we restrict ourselves here to the exponentially cutoff single powerlaw form:

$$
\phi(L) \propto\left(L / L_{p}\right)^{-\nu} e^{-L_{p} / L} .
$$

We assume that the GRB rate follows the SFR history, and consider two different SFR history pa- rameterizations which we label as follows. SFR1 is an update $(\mathrm{Li}$ 2008) of the SFR history models of Hopkins \& Beacom (2006) to include recent data from Bouwens et al. (2008) and Reddy et al. (2008), combined with a low-metallicity modification following the prescription of Langer \& Norman (2006). SFR2 is model A from Schmidt (2009) which represents a SFR history which remains constant beyond $z \sim 3$. It may, for example, be considered a more extreme lowmetallicity correction to the cosmic SFR, or represent a correction (Kistler et al. 2009; Virgili et al. 2011) to the high-redshift SFR as estimated from flux-limited surveys (by the integration of galaxy LFs thus obtained) due to a large amount of hidden star formation in faint, low-mass, and high specific SFR galaxies of the type that GRBs tend to be associated with at lower redshift (e.g. Fvnbo et al. 2002; Le Floc'h et al. 2003; Christensen et al. 2004; Fruchter et al. 2006).

Modeling is performed in the standard manner (e.g. Guetta \& Piran 2007) to produce $\log N-\log L$ number count distributions for various parameters of the LF, which are then fit by $\chi^{2}$ minimization to the observed $\log N-\log L$ distribution of all Swift bursts with peak photon flux $>1 \mathrm{~cm}^{-2} \mathrm{~s}^{-1}$. We emphasize that the redshift distribution is not part of this fitting procedure, but is always purely a result. In Fig. 18, we plot the redshift 
Table 2

Line Identifications, Observed EWs, and Fluxes

\begin{tabular}{|c|c|c|c|}
\hline GRB & Feature & $\begin{array}{c}\mathrm{EW}_{\mathrm{obs}} \\
(\AA)\end{array}$ & $\begin{array}{c}\text { Flux }\left(10^{-17}\right. \\
\left.\operatorname{erg~s}^{-1} \mathrm{~cm}^{-2}\right)\end{array}$ \\
\hline 050822 & {$[\mathrm{O}$ II] $\lambda 3727$} & $<-23$ & 2.2 \\
\hline \multirow{2}{*}{051006} & \begin{tabular}{l|l} 
O II & $\lambda 3727$
\end{tabular} & $-95.7 \pm 4.0$ & 7.5 \\
\hline & {$[\mathrm{Ne}$ III $] \lambda 3869$} & $-28.4 \pm 2.9$ & 2.0 \\
\hline \multirow{6}{*}{ 051117B } & 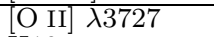 & $-12.5 \pm 1.5$ & 6.6 \\
\hline & $\mathrm{H} 10$ & $5.0 \pm 1.1$ & - \\
\hline & H9 & $6.8 \pm 1.1$ & - \\
\hline & $\mathrm{H} 8$ & $6.0 \pm 1.4$ & - \\
\hline & Ca II K & $5.2 \pm 1.1$ & - \\
\hline & $\mathrm{Ca}$ II $\mathrm{H}$ and $\mathrm{H} \varepsilon$ & - & - \\
\hline \multirow{2}{*}{$060805 \mathrm{~A}$} & {$[\mathrm{O}$ II $] \lambda 3727$} & $-20.0 \pm 4.0$ & 2.6 \\
\hline & [O III] $\lambda 5007$ & $-67.0 \pm 9.0$ & 7.6 \\
\hline \multirow{3}{*}{061021} & 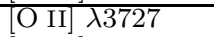 & $-49.1 \pm 9.9$ & 1.5 \\
\hline & {$\left[\begin{array}{lll}\text { O III] } & \lambda 4959\end{array}\right.$} & $-24.0 \pm 9.0$ & 0.8 \\
\hline & {$\left[\begin{array}{lll}\text { O III] } & \lambda 5007\end{array}\right.$} & $-51.0 \pm 9.5$ & 1.3 \\
\hline \multirow{2}{*}{070306} & O II $] \lambda 3726$ & $=$ & 170 \\
\hline & [O II] $\quad \lambda 3729$ & - & \\
\hline \multirow{2}{*}{070808} & {$[\mathrm{O}$ II $] \lambda 3727$} & $-65.3 \pm 5.1$ & 10.9 \\
\hline & $\mathrm{H} \beta$ & $-17.5 \pm 3.3$ & 4.5 \\
\hline
\end{tabular}

Note. - Flux measurement errors are of the order of $20 \%$. When the EW is not given, skylines prohibited a reliable estimate. The fluxes reported for GRB 060805A are those from object $A$. The fluxes reported for GRB 070808 are those from object $\mathrm{A}$. The flux in the emission line candidate in GRB 060923C is not reported due to the uncertainties introduced by the deconvolution.

distributions from our best fitting models in comparison to the TOUGH redshift data. The best fit model incorporating the SFR1 parameterization is a good fit to the number count distribution with a reduced $\chi^{2}=1.32$ (12 dof) and has parameters $L_{p}=10^{49.88} \mathrm{erg} \mathrm{s}^{-1}$ and $\nu=1.82$. The resultant redshift distribution is a good fit to the TOUGH redshift distribution up to $z \sim 1.5$, but underpredicts bursts at higher redshift. Overall, a Kolmogorov-Smirnov (KS) test yields a $<0.5 \%$ likelihood of the observed and model populations being the same. The SFR2 parameterization is a slightly better fit to the number count distribution with a reduced $\chi^{2}=1.30$ (12 dof) and LF parameters $L_{p}=10^{50.23} \mathrm{erg} \mathrm{s}^{-1}$ and $\nu=1.83$. The resultant redshift distribution in this case is an improved match to the overall observed TOUGH distribution with a KS likelihood of $42 \%$.

At face value, these results seem to imply that GRBs follow a cosmic SFR history that is significantly enhanced at high redshift compared to estimates from fluxlimited surveys. As previously discussed, given what is known about GRB hosts, it is entirely feasible that GRBs trace star formation at high redshift that would be undetectable by other means. It is of course also possible that the simple low-metallicity enhanced SFR parameterization used in the SFR 1 model is inadequate, or that the LF could have a more complex form and/or evolve with redshift.

An alternative approach to the modeling is to directly fit the observed joint peak-luminosity and redshift distribution of the sub-sample of TOUGH GRBs with redshifts. Again we take both SFR1 and SFR2, but in this case a broken power-law LF. We take a threshold flux for detection by the Burst Alert Telescope of $2 \times 10^{-8} \mathrm{erg} \mathrm{cm}^{-2} \mathrm{~s}^{-1}$, and apply a $K$-correction based on the observed spectral parameters for each burst, to bring the luminosity to a fixed restframe bandpass (30-
$300 \mathrm{keV}$ ). The results are very similar to the conclusions obtained above; hence we do not plot the inferred redshift distribution in Fig. 18. However, we do note that for SFR2 the maximum-likelihood solution gives the following LF parameters:

$$
\phi(L) \propto \begin{cases}L^{-1.52} ; & L<10^{52.5} \mathrm{erg} \mathrm{s}^{-1} \\ L^{-2.00} ; & L>10^{52.5} \mathrm{erg} \mathrm{s}^{-1} .\end{cases}
$$

\section{DISCUSSION AND IMPLICATIONS}

Figure 18 shows the cumulative redshift distribution of the largest homogeneous and unbiased sample of GRBs to date. It contains redshift information on all the 69 TOUGH bursts, including limits. The conservative systematic error region (hatched) of the TOUGH sample redshift distribution is significantly smaller than for previous samples (e.g. Figure 2 in Jakobsson et al. 2009). This allows the rejection of various model predictions (e.g. compare the dashed and dotted curves in Fig. 18). Thus, we have been able to confirm previous findings (e.g. Kistler et al. 2009; Virgili et al. 2011; Robertson \& Ellis $2012)$ that the GRB rate at high redshift $(z \gtrsim 3)$ appears to be in excess of predictions based on the assumption that it should follow conventional determinations of the star-formation history of the universe, combined with an estimate of its likely metallicity dependence (Langer \& Norman 2006).

It is possible that star formation at high redshifts has been significantly underestimated. Even at $z \sim$ 2 it appears that the galaxy LF has a substantially steeper faint-end slope than locally (e.g. Reddy \& Steidel 2009), while recent LF studies in the Hubble UltraDeep Field have concluded that at $z \gtrsim 7$ so-far undetected galaxies are likely to completely dominate the total star formation activity (e.g. Bouwens et al. 2011; Tanvir et al. 2012). This picture is supported by previous observations of damped $\operatorname{Ly} \alpha$ absorbers (e.g. Fynbo et al. 1999; Haehnelt et al. 2000; Schave 2001) and Ly $\alpha$ emitting galaxies (e.g. Fvnbo et al.|2003; Jakobsson et al. 2005), as well as by recent simulation studies (Choi \& Nagamine 2011). Alternatively, it could be that GRB production is substantially enhanced in the conditions of early star formation, beyond the metallicity-dependent rate correction already applied. In the long run, large complete samples of GRB redshifts should shed light on whether the GRB rate is proportional to SFR or whether other effects play an important role.

The sampling error and the conservative systematic error region are shown separately to clearly illustrate that incompleteness dominates the sample, and more is gained by reducing the systematics rather than increasing the sample size. Using both error regions we can set a conservative limit on the maximum number of Swift bursts at $z>6(z>7)$ : $14 \%(5 \%)$. This is fully consistent with the models which predict between 2 (SFR1) and 13 (SFR2) bursts per year (all sky) at $6<z<9$ to Swift/BAT limits.

The average (median) redshift of the 53 TOUGH bursts is $\langle z\rangle=2.23(\tilde{z}=2.14)$, significantly lower than the early Swift results indicated (e.g. Jakobsson et al. 2006). This difference may simply reflect the comparatively small samples analyzed in that paper, but could also be due to an increased success in measuring redshifts 


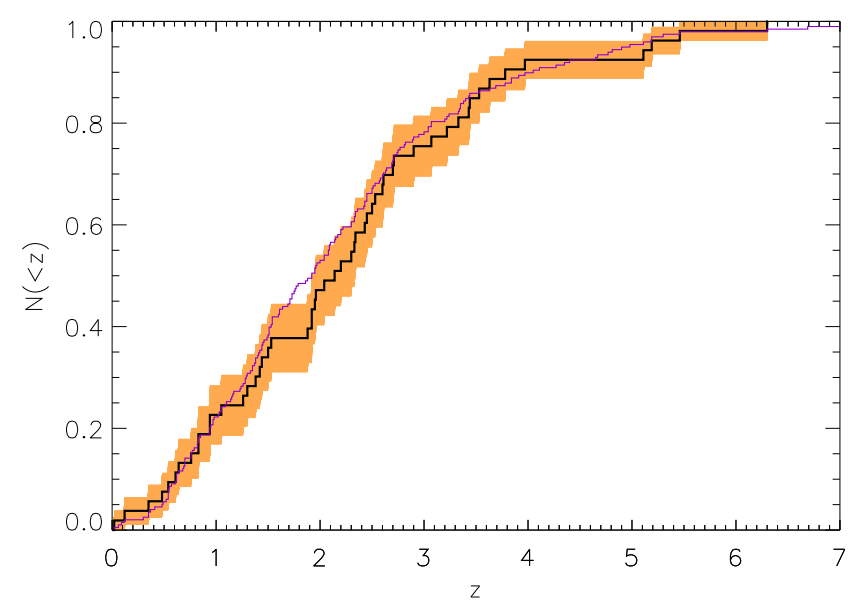

Figure 19. Thick solid curve and shaded region are defined as in Fig. 18 caption. The thin solid curve is the cumulative fraction of GRBs as a function of redshift for all long Swift bursts to date (2012 April 1: 198 redshifts) with $\langle z\rangle=2.16$.

$z<2$ using weaker absorption lines in afterglow spectra, and via host galaxies. The mean redshift could be as low as $\langle z\rangle \sim 1.7$ (upper boundary of the hatched region) although it is unlikely that the majority of bursts with unknown redshifts would be located at very small distances. In fact, it is more probable that $\langle z\rangle \gtrsim 2.20$ since we have only targeted the brightest galaxies in the sample $(R \lesssim 25 \mathrm{mag})$ for spectroscopic follow-up. In Fig. 19, we compare the TOUGH redshift distribution to the one obtained from all long-duration Swift bursts. The agreement is good in general but the TOUGH sample includes relatively more bursts in the range $1.8 \lesssim z \lesssim 2.5$. This can be explained by the fact that Ly $\alpha$ is located in the blue end of the optical at this redshift range which makes it easy to miss a damped Ly $\alpha$ absorber. On the other hand, emission lines are readily detectable with Xshooter at these redshifts.

As discussed in Hiorth et al. (2012), one of the TOUGH selection criteria (requiring the XRT error circle radius to be less than $2^{\prime \prime}$ ) might slightly bias our sample against faint, hence potentially high-redshift, bursts. In total, only two events were rejected due to a large error radius (GRBs 050412 and 061102). In both cases, the $\mathrm{X}$-ray afterglows were unusually faint, so this is an estimate of the fraction 14 (2/71) of Swift GRBs that would fail the TOUGH positional accuracy selection criterion which might well be at high redshift. Further to this, a comparable bias is introduced by the exclusion of bursts without a detected X-ray afterglow at all (5/180)15 All five GRBs are low-significance detections and are thus likely to be spurious. In total, a maximum of $5.5 \%$ of Swift long bursts could be at high redshift and not enter the TOUGH sample.

We have now reached a point in GRB research where a single burst rarely elucidates and illuminates our general understanding of the field. It is important to focus on well-defined samples and population studies, where systematics and biases can be minimized. Swift has made it possible to build such a sample and thanks to new

\footnotetext{
14 The total number of bursts here being the TOUGH 69 along with those two with a large error radius.

15 Swift detected 180 long bursts during the TOUGH time period in which the XRT was quickly $(<1 \mathrm{hr})$ repointed.
}

available instrumentation, such as the VLT/X-shooter (Vernet et al. 2011), we can continue to follow this track into the future.

We thank the referee for providing excellent comments in a timely manner and Andrew J. Levan for providing us with the NIR afterglow image for GRB 060814. We also thank Thomas Krühler, Dan Perley, and Ruben Salvaterra for very informative discussions. Based on observations collected at the European Southern Observatory, Chile, as part of the large program 177.A-0591. P.J. acknowledges support by a Project Grant from the Icelandic Research Fund. D.M. acknowledges financial support from the Instrument Center for Danish Astrophysics. The Dark Cosmology Centre is funded by the Danish National Research Foundation. This work has made use of the University of Hertfordshire Science and Technology Research Institute high-performance computing facility.

\section{REFERENCES}

Appenzeller, I., et al. 1998, The Messenger 94, 1

Bloom, J. S., Kulkarni, S. R., \& Djorgovski, S. G. 2002, AJ, 123, 1111

Bouwens, R. J., Illingworth, G. D., Franx, M., \& Ford, H. 2008, ApJ, 686, 230

Bouwens, R. J., et al. 2011, ApJ, in press (arXiv:1105.2038

Butler, N. R., Bloom, J. S., \& Poznanski, D. 2010, ApJ, 711, 495

Cao, X.-F., Yu, Y.-W., Cheng, K. S., \& Zheng, X.-P. 2011,

MNRAS, in press (arXiv:1101.0866)

Campana, S., Thöne, C. C., de Ugarte Postigo, A., Tagliaferri, G., Moretti, A., \& Covino, S. 2010, MNRAS, 402, 2429

Campisi, M. A., Li, L.-X., \& Jakobsson, P. 2010, MNRAS, 407, 1972

Chapman, R., Tanvir, N. R., Priddey, R. S., \& Levan, A. J. 2007, MNRAS, 382, L21

Choi, J.-H., \& Nagamine, K. 2012, MNRAS, 419, 1280

Christensen, L., Hjorth, J., \& Gorosabel, J. 2004, A\&A, 425, 913

Cobb, B. E., Bailyn, C. D., van Dokkum, P. G., \& Natarajan, P. 2006, ApJ, 645, L113

Courbin, F., Magain, P., Kirkove, M., \& Sohy, S. 2000, ApJ, 529, 1136

Coward, D. M. 2005, MNRAS, 360, L77

Coward, D. M., Guetta, D., Burman, R. R., \& Imerito, A. 2008, MNRAS, 386, 111

Daigne, F., Rossi, E. M., \& Mochkovitch, R. 2006, MNRAS, 372, 1034

Dall'Aglio, A., Wisotzki, L., \& Worseck, G. 2008, A\&A, 491, 465

Dong, Y.-M., \& Lu, T. 2009, RAA, 9, 95

Foley, S., McGlynn, S., Hanlon, L., McBreen, S., \& McBreen, B. 2008, A\&A, 484, 143

Fruchter, A. S., et al. 2006, Nature, 441, 463

Fynbo, J. P. U., Møller, P., \& Warren, S. J. 1999, MNRAS, 305, 849

Fynbo, J. P. U., et al. 2002, A\&A, 388, 425

Fynbo, J. P. U., Ledoux, C., Møller, P., Thomsen, B., \& Burud, I. 2003, A\&A, 407, 147

Fynbo, J. P. U., Prochaska, J. X., Sommer-Larsen, J.,

Dessauges-Zavadsky, M., \& Møller, P. 2008, ApJ, 683, 321

Fynbo, J. P. U., et al. 2009, ApJS, 185, 526

Gehrels, N., et al. 2004, ApJ, 611, 1005

Gorosabel, J., Lund, N., Brandt, S., Westergaard, N. J., \& Castro Cerón, J. M. 2004, A\&A, 427, 87

Grupe, D., et al. 2007, AJ, 133, 2216

Guetta, D., Piran, T., \& Waxman, E. 2005, ApJ, 619, 412

Guetta, D., \& Piran, T. 2007, JCAP, 7, 3

Haehnelt, M. G., Steinmetz, M., \& Rauch, M. 2000, ApJ, 534, 594

Hjorth, J., et al. 2003, Nature, 423, 847

Hjorth, J., et al. 2012, ApJ, submitted

Hopkins, A. M., \& Beacom, J. F. 2006, ApJ, 651, 142

Jakobsson, P., Hjorth, J., Fynbo, J. P. U., Watson, D., Pedersen, K., Björnsson, G., \& Gorosabel, J. 2004, ApJ, 617, L21

Jakobsson, P., et al. 2005, MNRAS, 362, 245

Jakobsson, P., et al. 2006, A\&A, 447, 897

Jakobsson, P., Malesani, D., Fynbo, J. P. U., Hjorth, J., \&

Milvang-Jensen, B. 2009, in AIP Conference Proceedings 1133,

Gamma-Ray Bursts: Sixth Huntsville Symposium, ed. C.

Meegan, N. Gehrels, \& C. Kouveliotou (New York: AIP), 455 
Jakobsson, P., Malesani, D., Hjorth, J., Fynbo, J. P. U., \& Milvang-Jensen, B. 2011a, AdSpR, 47, 1416

Jakobsson, P., Malesani, D., Hjorth, J., Fynbo, J. P. U., \& Milvang-Jensen, B. 2011b, AN, 332, 276

Jakobsson, P., Malesani, D., Hjorth, J., Fynbo, J. P. U., \& Milvang-Jensen, B. 2011c, in AIP Conference Proceedings 1358 Gamma Ray Bursts 2010, ed. J. E. McEnery, J. L. Racusin \& N. Gehrels (New York: AIP), 265

Jaunsen, A. O., et al. 2008, ApJ, 681, 453

Jarosik, N., et al. 2011, ApJS, 192, 14

Kistler, M. D., Yüksel, H., Beacom, J. F., \& Stanek, K. Z. 2008 , ApJ, 673, L119

Kistler, M. D., Yüksel, H., Beacom, J. F., Hopkins, A. M., \& Wyithe, J. S. B. 2009, ApJ, 705, L104

Krühler, T., et al. 2012, ApJ, submitted

Langer, N., \& Norman, C. A. 2006, ApJ, 638, L63

Le, T., \& Dermer, C. D. 2007, ApJ, 661, 394

Le Floc'h, E., et al. 2003, A\&A, 400, 499

Levan, A. J., Tanvir, N. R., Rol, E., Fruchter, A., \& Adamson, A. 2006, GCN Circ. 5455

Letawe, Y., Magain, P., Letawe, G., Courbin, F., \& Hutsemékers, D. 2008, ApJ, 679, 967

Levesque, E. M., Kewley, L. J., Berger, E., \& Jabran Zahid, H. 2010, AJ, 140,1557

Li, L.-X. 2008, MNRAS, 388, 1487

Liang, E., Zhang, B., Virgili, F., \& Dai, Z. G. 2007, ApJ, 662, 1111

Malesani, D. 2006, GCN Circ. 5456

Malesani, D., et al. 2012, ApJ, in preparation

Milvang-Jensen, B., et al. 2012, ApJ, submitted

Natarajan, P., Albanna, B., Hjorth, J., Ramirez-Ruiz, E., Tanvir, N., \& Wijers, R. 2005, MNRAS, 364, L8

Ovaldsen, J.-E., et al. 2007, ApJ, 662, 294

Paczyński, B. 1998, ApJ, 494, L45

Perley, D. A., et al. 2009, AJ, 138, 1690

Porciani, C., \& Madau, P. 2001, ApJ, 548, 522
Predehl, P., \& Schmitt, J. H. M. M. 1995, A\&A, 293, 889

Reddy, N. A., \& Steidel, C. C. 2009, ApJ, 692, 778

Reddy, N. A., Steidel, C. C., Pettini, M., Adelberger, K. L. Shapley, A. E., Erb, D. K., \& Dickinson, M. 2008, ApJS, 175, 48

Robertson, B. E., \& Ellis, R. S. 2012, ApJ, 744, 95

Rol, E., Jakobsson, P., Tanvir, N., \& Levan, A. 2006, GCN Circ. 5555

Salvaterra, R., \& Chincarini, G. 2007, ApJ, 656, L49

Salvaterra, R., Campana, S., Chincarini, G., Tagliaferri, G., \& Covino, S. 2007, MNRAS, 380, L45

Salvaterra, R., Guidorzi, C., Campana, S., Chincarini, G., \&

Tagliaferri, G. 2009, MNRAS, 396, 299

Salvaterra, R., et al. 2012, ApJ, 749, 68

Savaglio, S., Glazebrook, K., \& Le Borgne, D. 2009, ApJ, 691, 182

Schaye, J. 2001, ApJ, 559, L1

Schmidt, M. 2009, ApJ, 700, 641

Soderberg, A. M., et al. 2006, Nature, 442, 1014

Songaila, A. 2004, AJ, 127, 2598

Tanvir, N. R., \& Jakobsson, P. 2007, RSPTA, 365, 1377

Tanvir, N. R., et al. 2008, MNRAS, 388, 1743

Tanvir, N. R., et al. 2012, ApJ, in press (arXiv:1201.6074)

Thöne, C. C., Perley, D. A., \& Bloom, J. S. 2007, GCN Circ. 6663

Vernet, J., et al. 2011, A\&A, 536, 105

Virgili, F. J., Liang, E.-W., \& Zhang, B. 2009, MNRAS, 392, 91

Virgili, F. J., Zhang, B., Nagamine, K., \& Choi, J.-H. 2011, MNRAS, 417, 3025

van Dokkum, P. G. 2001, PASP, 113, 1420

Wanderman, D. \& Piran, T. 2010, MNRAS, 406, 1944

Wijers, R. A. M. J., Bloom, J. S., Bagla, J. S., \& Natarajan, P. 1998, MNRAS, 294, L13

Woosley, S. E., \& Bloom, J. S. 2006, ARA\&A, 44, 507

Zitouni, H., Daigne, F., Mochkovich, R., \& Zerguini, T. H. 2008, MNRAS, 386, 1597 